\title{
Highly active manganese oxide catalysts for low-temperature oxidation of formaldehyde
}

\author{
Hua Tian ${ }^{a}$, Junhui He ${ }^{\mathrm{a}, *}$, Linlin Liu ${ }^{\mathrm{a}}$, Donghui Wang ${ }^{\mathrm{b}}$, Zhengping Hao ${ }^{\mathrm{c}}$, Chunyan Ma ${ }^{\mathrm{c}}$ \\ a Functional Nanomaterials Laboratory, Technical Institute of Physics and Chemistry, Chinese Academy of Sciences, Beijing 100190, China \\ ${ }^{\mathrm{b}}$ Research Institute of Chemical Defence, Beijing 100083, China \\ ${ }^{\mathrm{c}}$ Department of Environmental Nano-materials, Research Center for Eco-Environmental Sciences, Chinese Academy of Sciences, Beijing 100085, China
}

\section{A R T I C L E I N F O}

\section{Article history:}

Received 29 July 2011

Received in revised form 27 September 2011

Accepted 5 October 2011

Available online 19 October 2011

\section{Keywords:}

Manganese oxide

Birnessite

Formaldehyde

Complete oxidation

\begin{abstract}
A B S T R A C T
Birnessite - type manganese oxides with very high surface areas (up to $154 \mathrm{~m}^{2} / \mathrm{g}$ ) were successfully prepared using a microemulsion process. The morphology, surface area, pore size, and the surface reducibility of these materials were readily tailored via the synthesis temperature. The physiochemical properties of the manganese oxides were characterized by means of XRD, TEM, SEM, BET and $\mathrm{H}_{2}$-TPR techniques. These materials were also catalytically tested in HCHO oxidation, showing significantly high catalytic activity. It was found that the differences in catalytic performance of these materials were jointly attributed to the effects of the morphology, surface area and surface reducibility. The highly porous feature of the catalyst with nanospheres could allow $\mathrm{HCHO}$ molecules to easily diffuse onto catalytically active sites, which endows the nanospheres with high catalytic activity. The higher the surface area, the higher its catalytic activity. A higher surface reducibility offered a lower temperature of $\mathrm{HCHO}$ oxidation. The most active manganese catalyst (BSW-120) showed a $100 \% \mathrm{HCHO}$ conversion at $100{ }^{\circ} \mathrm{C}$.
\end{abstract}

(c) 2011 Elsevier Inc. All rights reserved.

\section{Introduction}

Due to stringent environmental regulations for human health protection, low-temperature catalytic oxidation of formaldehyde ( $\mathrm{HCHO})$ to $\mathrm{CO}_{2}$ and $\mathrm{H}_{2} \mathrm{O}$ has become an attractive research topic [1-5]. In the past decades, a variety of catalysts, including metal oxides [3,6], composite oxides [7], and supported noble metals [8] have been investigated for the $\mathrm{HCHO}$ emission control. Among them, catalysts made of noble metals ( $\mathrm{Au}, \mathrm{Ag}$, Pd, and $\mathrm{Pt}$ ) supported on metal oxides have been proved to be highly active at relatively low reaction temperatures. For instance, Zhang et al. [9] observed a $100 \% \mathrm{HCHO}$ conversion at $\sim 75^{\circ} \mathrm{C}$ over three-dimensionally ordered macroporous $\mathrm{Au} / \mathrm{CeO}_{2}$ catalysts. An et al. [10] found that $\mathrm{Pt} / \mathrm{Fe}_{2} \mathrm{O}_{3}$ prepared by a colloid deposition route performed highly active for HCHO oxidation at ambient temperature. However, the scarcity and high cost of these noble metals have long motivated the research for substitute catalysts. Recently, considerable efforts have been devoted to the design of catalysts based on transition metal oxides, like $\mathrm{MnO}_{x}$, which exhibit catalytic activity for $\mathrm{HCHO}$ oxidation as high as or even slightly higher than those of the supported noble metals $[3,11]$.

\footnotetext{
* Corresponding author. Tel./fax: +86 1082543535

E-mail address: jhhe@mail.ipc.ac.cn (J. He).
}

Layered birnessite manganese oxides, as environmentalfriendly materials, have been extensively studied for the catalytic reduction of $\mathrm{NO}_{x}$ [12], decomposition of phenol [13], or the catalytic combustion of volatile organic compounds (VOCs) [14] because of their high catalytic activity and low cost. We recently reported that birnessite catalysts exhibited excellent catalytic activity for the complete oxidation of $\mathrm{HCHO}$ at relatively low temperatures [15]. It is well-known that catalytic activities are associated with the surface area, pore size and transition metal ion redox ability (surface reducibility) of the catalyst. Although a considerable number of strategies have been explored for tailoring their morphology, particle size, and framework substitution [16-19], to the best of our knowledge, there have been reported few birnessite materials, especially crystalline K-type birnessite materials with very high surface areas $\left(>100 \mathrm{~m}^{2} / \mathrm{g}\right)$. In a very recent work, we reported a novel microemulsion method for synthesis of birnessite manganese oxides [20]. Well-crystallized birnessite materials with varied morphologies and crystalline phases were obtained. Among them, a novel morphology of acanthosphere shape has a large specific surface area, and would be very promising for catalytic decomposition of HCHO.

In this work, we optimized the synthesis conditions, investigated the catalytic activity for $\mathrm{HCHO}$ oxidation, and found it is in fact the case, i.e., the acanthosphere birnessite manganese oxide showed excellent catalytic activity for $\mathrm{HCHO}$ decomposition. 


\section{Experimental}

\subsection{Synthesis of catalysts}

In a typical synthesis, $23 \mathrm{~g}$ of benzyl alcohol (BA) and $12.32 \mathrm{~g}$ sodium dodecyl sulfate (SDS) were dissolved in $64.68 \mathrm{~g}$ of distilled water under vigorous stirring. The weight composition of BA/SDS/ $\mathrm{H}_{2} \mathrm{O}$ in the system was 30:16:84. 3.16 $\mathrm{g}$ of $\mathrm{KMnO}_{4}$ was then added in the solution. The reaction proceeded with the appearance of a brown precipitate within a few minutes. After stirring at room temperature for $0.5 \mathrm{~h}$, the mixture was heated at $80^{\circ} \mathrm{C}$ for $2 \mathrm{~h}$ in a Teflon lined autoclave. The resulting product was collected, and washed several times with distilled water and absolute alcohol, respectively. Finally, the product was dried overnight at $60^{\circ} \mathrm{C}$, and labeled as BSW-80. Manganese oxide products obtained by heating at 100 , 120 and $140{ }^{\circ} \mathrm{C}$ under otherwise identical conditions are denoted as BSW-100, BSW-120 and BSW-140, respectively.

\subsection{Characterization of catalysts}

The structure of the prepared materials was first analyzed with X-ray diffraction (XRD) using a Bruker D8 Focus diffractometer with a $\mathrm{Cu} \mathrm{K} \alpha \mathrm{X}$-ray source $(40 \mathrm{~mA}, 40 \mathrm{kV})$. The thermal stability was accessed by thermogravimetric analysis and differential thermal analysis (TGA-DTA), which were performed on a SDT Q600 thermal gravimetric analyzer in dry air with a heating rate of $10{ }^{\circ} \mathrm{C} / \mathrm{min}$. The morphologies of the products were studied by transmission electron microscopy (TEM) on a JEOL JEM-2100F electron microscope. Scanning electron microscopy (SEM) images were obtained on a Hitachi S-4300 electron microscope operating at $10 \mathrm{kV}$. The Braunauer-Emmett-Teller (BET) surface area measurements were carried out at $77 \mathrm{~K}$ using a Quadrasorb SI automated surface area and pore size analyzer. Hydrogen temperature-programmed reduction $\left(\mathrm{H}_{2}\right.$-TPR) was carried out in a microreactor using $50 \mathrm{mg}$ of sample for each run. At constant temperature of $50{ }^{\circ} \mathrm{C}$, the flow of $\mathrm{H}_{2} / \mathrm{Ar}\left(5 \% \mathrm{H}_{2}, 30 \mathrm{~mL} / \mathrm{min}\right)$ was switched to the sample, and after $30 \mathrm{~min}$ the temperature-programmed reduction was started from $50{ }^{\circ} \mathrm{C}$ to $900{ }^{\circ} \mathrm{C}$ with a temperature ramp of $5{ }^{\circ} \mathrm{C} \mathrm{min}^{-1}$. The consumption of the reducing agent $\mathrm{H}_{2}$ was measured on-line by a gas chromatograph equipped with a TCD.

\subsection{Catalytic test}

Catalytic activities were evaluated in a fixed-bed reactor under atmospheric pressure. The catalyst (100 mg, 40-60 mesh) was loaded in a quartz tube reactor (i.d. $=4 \mathrm{~mm}$ ). Gaseous $\mathrm{HCHO}$ was generated by passing a purified air flow over HCHO solution in an incubator kept at $0{ }^{\circ} \mathrm{C}$, leading to a feed gas with $460 \mathrm{ppm}$ of $\mathrm{HCHO}$. The total flow rate was $50 \mathrm{~mL} / \mathrm{min}$ in a space velocity of $30,000 \mathrm{~mL} /\left(g_{\text {cat }} \mathrm{h}\right)$. The effluents from the reactor were analyzed with an on-line Agilent 6890 gas chromatograph equipped with FID and Ni catalyst converter which was used for converting carbon oxides quantitatively into methane in the presence of hydrogen before the detector. No other carbon containing compounds except $\mathrm{CO}_{2}$ in the products were detected for all tested catalysts. Thus, $\mathrm{HCHO}$ conversion was expressed in the yield of $\mathrm{CO}_{2}$, and calculated as follows:

$\mathrm{HCHO}$ conversion $(\%)=\mathrm{CO}_{2}$ yield $(\%)=\frac{\left[\mathrm{CO}_{2}\right]_{\text {out }} \text { vol. } \%}{[\mathrm{HCHO}]_{\text {in }} \text { vol. } \%} \times 100$

where $\left[\mathrm{CO}_{2}\right]_{\text {out }}$ was the $\mathrm{CO}_{2}$ concentration in the products (vol.\%), and $[\mathrm{HCHO}]_{\text {in }}$ was the $\mathrm{HCHO}$ concentration of the feed gas (vol.\%).

\section{Results and discussion}

\subsection{Features of materials}

The XRD patterns of as-prepared manganese oxide materials are displayed in Fig. 1, and confirm the formation of K-birnessite phase [21]. Broad peaks in Fig. 1a relate to the poorly-crystallized nature of the sample. When the synthesis temperature rises to $100^{\circ} \mathrm{C}$, the XRD peak intensities increase greatly (Fig. 1b and c). Clearly, transformation occurs from the poorly-crystallized initial aggregates into a well-crystallized structure. Further increase of the temperature to $140{ }^{\circ} \mathrm{C}$ would decompose part of the formed birnessite to $\mathrm{Mn}_{3} \mathrm{O}_{4}$ (Fig. 1d), which is due to the excessive reduction of $\mathrm{Mn}^{7+}$ by BA. The reduction ability of BA was previously utilized by Xiao et al. and our group to synthesize metal oxides [20,22]. It was reported that higher temperature would lead to a higher reduction ability of BA, and more $\mathrm{Mn}^{7+}$ ions were reduced to $\mathrm{Mn}$ in lower oxidation states such as $\mathrm{Mn}^{2+}$ and $\mathrm{Mn}^{3+}$.

The TGA-DTA measurement of a selected manganese oxide sample is shown in Fig. 2. The profile reveals three weight losses in the temperature range of $25-800^{\circ} \mathrm{C}$. (i) The first weight loss of $11.0 \%$ in the range of $25-205^{\circ} \mathrm{C}$ is attributed to physisorbed and interlayer water in birnessite nanostructure. (ii) The second weight loss of $2.9 \%$ in the $205-510{ }^{\circ} \mathrm{C}$ range is slightly complicated. In the $205-260{ }^{\circ} \mathrm{C}$ range, an exothermic peak appears at ca. $233^{\circ} \mathrm{C}$ on the DTA curve, while the weight loss is not significant. This likely corresponds to the decomposition of tiny residual SDS in birnessite pores. The weight loss in the range of $260-510^{\circ} \mathrm{C}$ is related to the oxygen release due to the partial reduction of the $\mathrm{Mn}^{4+}$ cations to their trivalent state [23]. (iii) Above $510^{\circ} \mathrm{C}$, the gained weight is possibly due to the compensation of released oxygen produced by the oxidation of $\mathrm{Mn}^{3+}$ to $\mathrm{Mn}^{4+}$, which leads to the phase transformation from the layered structure to the $\alpha-\mathrm{MnO}_{2}$ phase [23]. The exothermic peak at $533{ }^{\circ} \mathrm{C}$ is related to this phase transformation. In addition, below $250^{\circ} \mathrm{C}$, no significant endothermic peak and weight loss of BA (its boiling point is $205^{\circ} \mathrm{C}$ ) appears, suggesting that BA should have been removed during the synthesis process.

The nanostructures of manganese oxides prepared at different temperatures were observed using TEM and SEM. BSW-80 shows agglomerated cotton-like nanospheres that consist of ultrafine crystals (Figs. 3a and 4a). It is poorly-crystallized, as was confirmed by the XRD analysis. When the temperature rises to $100{ }^{\circ} \mathrm{C}$, flake architectures are represented with some twisting fiber-like manner (Figs. 3b and $4 \mathrm{~b}$ ). It is interesting to learn that when up to $120^{\circ} \mathrm{C}$, in addition to the flakes, some nanospheres of ca. $500 \mathrm{~nm}$

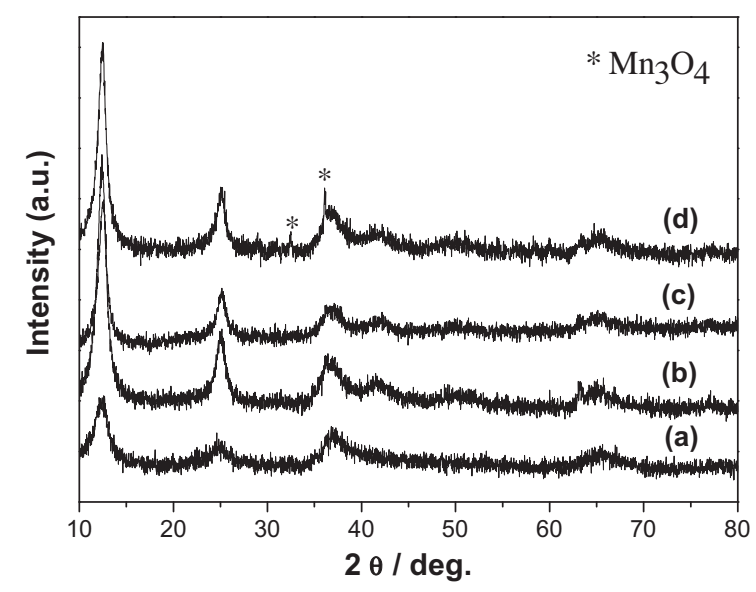

Fig. 1. XRD patterns of varied manganese oxide catalysts: (a) BSW-80, (b) BSW-100, (c) BSW-120, and (d) BSW-140 


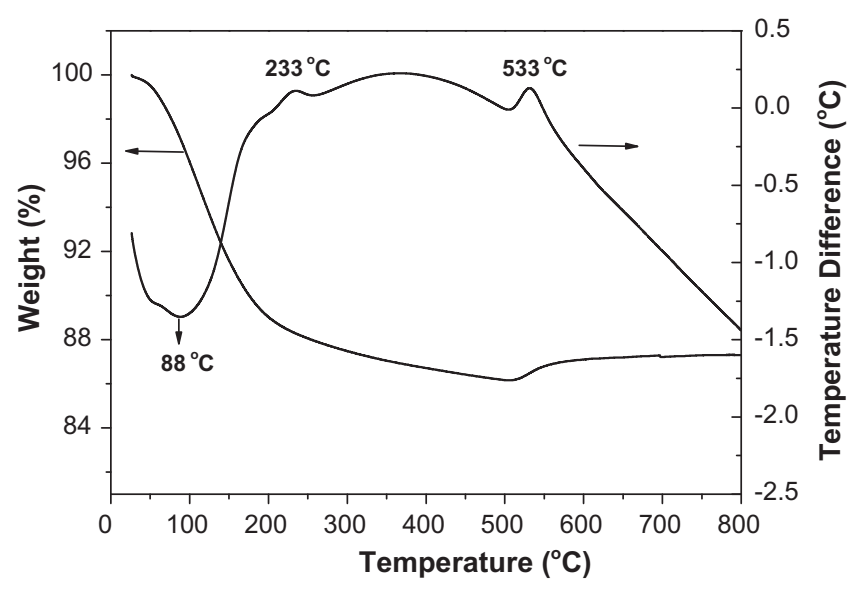

Fig. 2. TG and DTA curves of BSW-120 catalyst.

appear, which are probably assembled from smaller flakes. The widening of the XRD peaks (Fig. $1 \mathrm{~b}$ and $\mathrm{c}$ ) supports this speculation. With further increasing the synthesis temperature to $140{ }^{\circ} \mathrm{C}$, however, the nanospheres disappear, and a novel nano-octahedra structure is observed (Fig. $3 \mathrm{~d}$, pointed by arrow). This nanooctahedra structure corresponds to the $\mathrm{Mn}_{3} \mathrm{O}_{4}$ phase [24], in accordance with those revealed by XRD for BSW-140.

It is well known that microemulsion systems have different phase structures such as lamellar liquid crystal region $\left(L_{c}\right)$, hexagonal liquid crystal region (Hex) and isotropic liquid regions $\left(L_{1}, \mathrm{o} / \mathrm{w}\right.$ structure and $L_{2}, \mathrm{w} / \mathrm{o}$ structure). Among them, $L_{1}$ region consists of three subregions: rod, spherical and bicontinuous regions. These structures can be obtained by changing the amount of each component [25]. Xu and Liu [26] reported that in the $\mathrm{SDS} / \mathrm{BA} / \mathrm{H}_{2} \mathrm{O}$ system, when the weight ratio of $\mathrm{SDS} / \mathrm{H}_{2} \mathrm{O}$ was $16: 84$, the structure of system appeared as rod (BA $<1.0 \mathrm{wt} \%)$, spherical (1.0 wt\% $<\mathrm{BA}<16.0$ wt\%) and bicontinuous (BA $>16.0 \mathrm{wt} \%$ ), respectively. It is evident from these results that the composition used the current work $\left(\mathrm{SDS} / \mathrm{H}_{2} \mathrm{O}=16: 84, \mathrm{BA}=23 \mathrm{wt} \%\right)$ should exhibit the bicontinuous structure. It is further noted that in the bicontinuous structure, both water and BA are the solubilized phase and continuous phase, simultaneously, and the SDS aggregate shows a network structure. Hence, cotton-like nanospheres and flake architectures of manganese oxides are obtained when the synthesis temperatures are not too high. While the synthesis temperature rises to $120^{\circ} \mathrm{C}, \mathrm{BA}$, which was used as a microemulsion component and reducing agent, simultaneously, may be consumed faster than that at lower temperature, leading to the formation of spherical region in microemulsion. In this case, nanosphere-like manganese oxide appears. However, further increasing the synthesis temperature to $140{ }^{\circ} \mathrm{C}$ will destroy the microemulsion system [27], leading to the formation of scattered flake manganese oxide again.

Fig. 5 shows the $\mathrm{N}_{2}$ adsorption isotherms of manganese oxide materials. There is no clear capillary condensation and hysteresis loop at high $p / p_{0}$ for the BSW-80 sample, indicating its poorly porous nature. In contrast, type II adsorption isotherms with capillary condensation at high $p / p_{0}$ can be observed for BSW-100, BSW-120 and BSW-140, respectively. A hysteresis loop of type H3, according to the IUPAC classification of types of hysteresis loops [28], occurs at high $p / p_{0}$ close to saturation, suggesting the existence of slitshaped mesopores with nonuniform sizes, probably due to the aggregation of nanoparticles. BSW-100 adsorbs apparently more nitrogen molecules than other products, indicating its larger pore
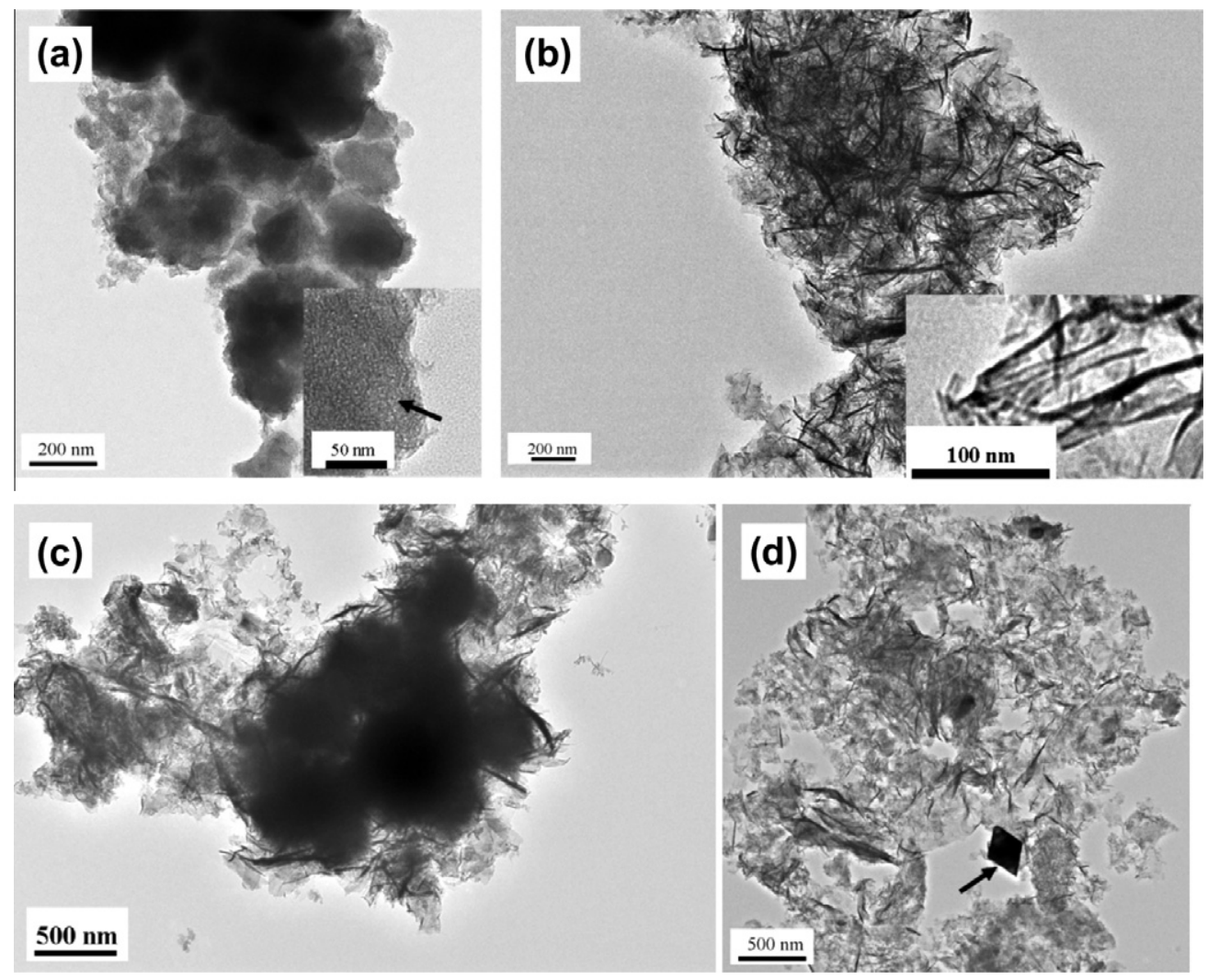

Fig. 3. TEM images of varied manganese oxide catalysts: (a) BSW-80, inset: HRTEM image, (b) BSW-100, inset: HRTEM image, (c) BSW-120, and (d) BSW-140. 

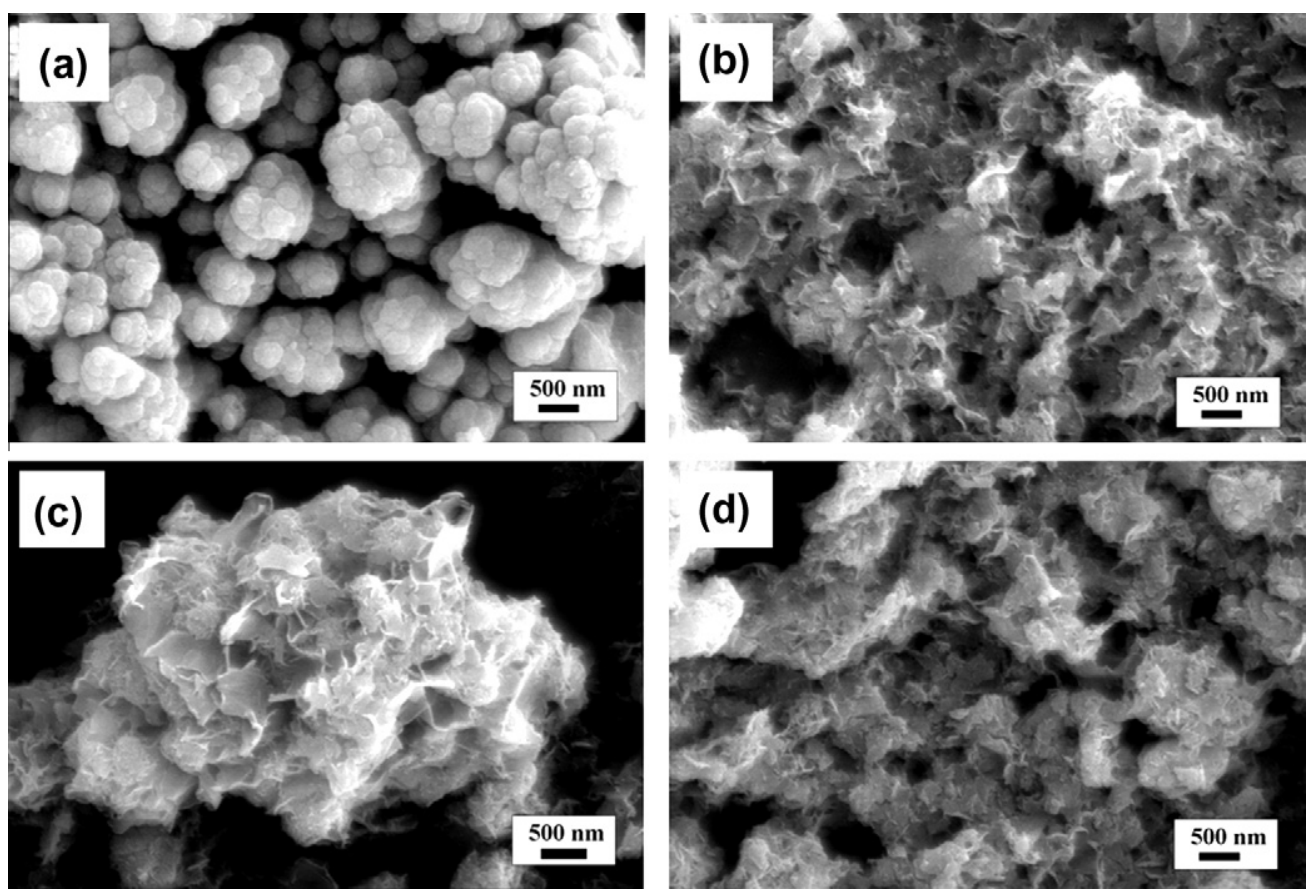

Fig. 4. SEM images of varied manganese oxide catalysts: (a) BSW-80, (b) BSW-100, (c) BSW-120, and (d) BSW-140.

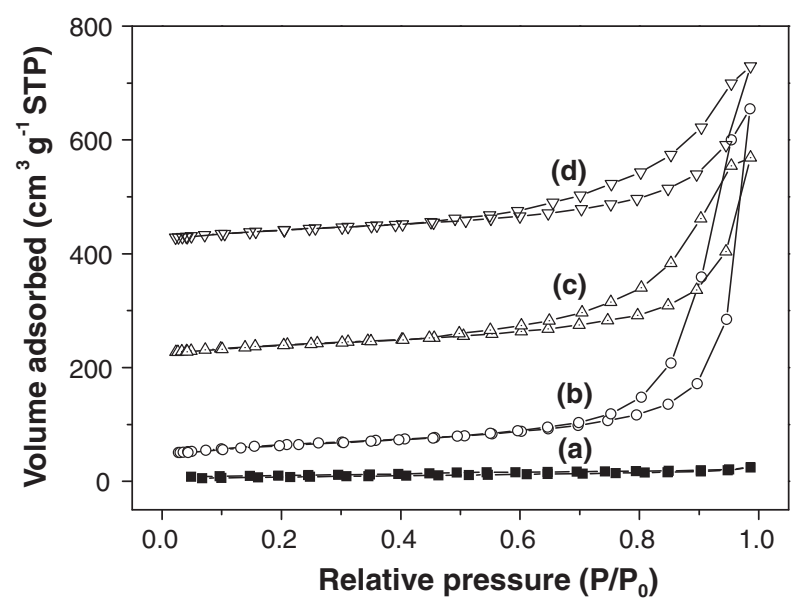

Fig. 5. $\mathrm{N}_{2}$ adsorption-desorption isotherms of varied manganese oxide catalysts: (a) BSW-80, (b) BSW-100, (c) BSW-120, and (d) BSW-140.

volumes. But the hysteresis loop of BSW-100 is also relatively broader, which demonstrates that the bottle-neck effect of the BSW-100 pores becomes much more evident.
All the textural properties of the as-prepared materials are summarized in Table 1 . The BET surface area and total pore volume of BSW-80 is $28 \mathrm{~m}^{2} / \mathrm{g}$ and $0.039 \mathrm{~cm}^{3} / \mathrm{g}$ respectively, much lower than those of BSW-100, BSW-120 and BSW-140. These results match the XRD (Fig. 1) and $\mathrm{N}_{2}$ adsorption isotherm (Fig. 5) data. The nearly amorphous nature of BSW-80 leads to its poor pore structure, and eventually to the low surface area and pore volume. BSW100 has the highest surface area $\left(154 \mathrm{~m}^{2} / \mathrm{g}\right)$ and the largest pore volume $\left(0.984 \mathrm{~cm}^{3} / \mathrm{g}\right)$. The BET surface areas of BSW-120 and BSW-140 are 138 and $145 \mathrm{~m}^{2} / \mathrm{g}$, respectively, lower than that of BSW-100 but still much higher than those of reported for crystallized K-type birnessite to date. These results are consistent with the TEM observations (Fig. 3). BSW-100 consists of well-crystallized flakes, whereas some nanospheres and nano-octahedra appear in BSW-120 and BSW-140, respectively, all of which are accompanied by a decrease in the surface area.

According to Table 1, the micropores only contribute $1-2 \%$ of total pore volume. The most probable pore size in the micropore range is around $0.6 \mathrm{~nm}$. Compared to $\mathrm{BSW}-80$, the other three products prepared at higher temperatures have larger most probable mesopore sizes (14-26 nm, compared to $5.4 \mathrm{~nm}$ of BSW-80) and pore volumes. The mesopore size and pore volume of BSW100 is found to be the highest among others, as well as its surface area.

Table 1

Textural properties and catalytic performance of manganese oxide catalysts.

\begin{tabular}{|c|c|c|c|c|c|c|c|c|}
\hline \multirow[t]{2}{*}{ Sample } & \multirow[t]{2}{*}{$S_{\mathrm{BET}}{ }^{\mathrm{a}}\left(\mathrm{m}^{2} / \mathrm{g}\right)$} & \multicolumn{2}{|c|}{ Pore size $(\mathrm{nm})$} & \multicolumn{2}{|c|}{ Pore volume $\left(\mathrm{cm}^{3} / \mathrm{g}\right)$} & \multirow[t]{2}{*}{$\% V_{\text {mic }}$} & \multicolumn{2}{|c|}{ HCHO oxidation activity $\left({ }^{\circ} \mathrm{C}\right)$} \\
\hline & & $\overline{D_{\text {mic }}{ }^{b}}$ & $D_{\text {mes }}{ }^{\mathrm{c}}$ & $\overline{V_{\text {mic }}{ }^{\mathrm{d}}}$ & $V_{\text {total }} \mathrm{e}$ & & $\overline{T_{30}}$ & $T_{50}$ \\
\hline BSW-80 & 28 & 0.6 & 5.4 & 0 & 0.039 & 0 & 83 & 100 \\
\hline BSW-100 & 154 & 0.6 & 25.6 & 0.013 & 0.984 & 1.3 & 77 & 92 \\
\hline BSW-120 & 138 & 0.6 & 16.6 & 0.007 & 0.572 & 1.2 & 68 & 83 \\
\hline BSW-140 & 145 & 0.5 & 14.1 & 0.010 & 0.511 & 2.0 & 64 & 86 \\
\hline
\end{tabular}

a Total surface area obtained by the BET method.

b The most probable micropore size based on the HK method.

c The most probable mesopore size based on the BJH method.

d Micropore ( $<2 \mathrm{~nm}$ ) volume calculated using the t-plot method.

e Total pore volumes obtained by $\mathrm{N}_{2}$ adsorption at $p / p_{0}=0.99$. 


\subsection{Activity test}

Results for the HCHO oxidation over the manganese oxide catalysts prepared at varied temperatures are shown in Fig. 6. In all cases, in order to check for the complete oxidation of $\mathrm{HCHO}$ as accurately as possible, only $\mathrm{CO}_{2}$ was detected as the oxidation product of $\mathrm{HCHO}$. It is noted that $\mathrm{CO}_{2}$ yield increases with the rise of oxidation temperature over the catalysts. Among all the catalysts tested, BSW-120 gives the highest catalytic activity. A $100 \%$ yield of $\mathrm{CO}_{2}$ over BSW-120 was achieved at $100^{\circ} \mathrm{C}$ which is much lower as compared to other manganese oxides reported elsewhere under similar conditions $[3,11,29,30]$, indicating that the porous manganese oxide BSW-120 is an excellent catalyst for $\mathrm{HCHO}$ oxidation. These catalysts should measure even better when the $\mathrm{HCHO}$ conversion is determined by the decrease of $\mathrm{HCHO}$ concentration, as reported in some literatures [15]. Because the conversion obtained by this method includes the amount of adsorbed $\mathrm{HCHO}$ during the catalytic reaction, and thus gives a conversion probably higher than the real value.

The temperatures of $30 \% \mathrm{CO}_{2}$ yield $\left(T_{30}\right)$ and $50 \% \mathrm{CO}_{2}$ yield $\left(T_{50}\right)$ over different catalysts are presented in Table 1 . The $T_{50}$ decreases greatly with increasing the synthesis temperature from 80 to $120^{\circ} \mathrm{C}$. However, further increasing the temperature (from 120 to $140{ }^{\circ} \mathrm{C}$ ) leads to the slight decrease of the catalytic activity. Moreover, it is interesting that BSW-140 with $T_{30}$ of $64^{\circ} \mathrm{C}$ shows slightly higher activity than BSW-120 with $T_{30}$ of $68^{\circ} \mathrm{C}$, as shown in Fig. 6. Clearly, the discrepancies in the structural and chemical properties of these catalysts greatly affect the catalytic behavior.

The porous structure of the catalyst may facilitate the diffusion of the HCHO molecules. An earlier study on VOCs oxidation revealed that high surface area is much crucial for high catalytic activity of catalyst [31,32]. It is mentioned above that BSW-80 consists of agglomerated nanospheres, showing much lower surface area than those of BSW-100, BSW-120 and BSW-140, and thus naturally has a lower activity. BSW-100 and BSW-120 have comparable surface areas, but compared with BSW-100, BSW-120 has smaller pore size and consists of some nanospheres of ca. $500 \mathrm{~nm}$, which could prolong the stay time of HCHO molecules in the catalyst, and enhance $\mathrm{HCHO}$ oxidation. Our previous work [15] also showed that the morphology largely affected on the catalytic activity of $\mathrm{MnO}_{2}$ nanomaterials. Hollow $\mathrm{K}_{x} \mathrm{MnO}_{2}$ nanospheres exhibited larger catalytic activity than honeycomb $\mathrm{K}_{x} \mathrm{MnO}_{2}$ nanospheres because the former could adsorb and retain $\mathrm{HCHO}$ for a longer period of time than the latter, and eventually

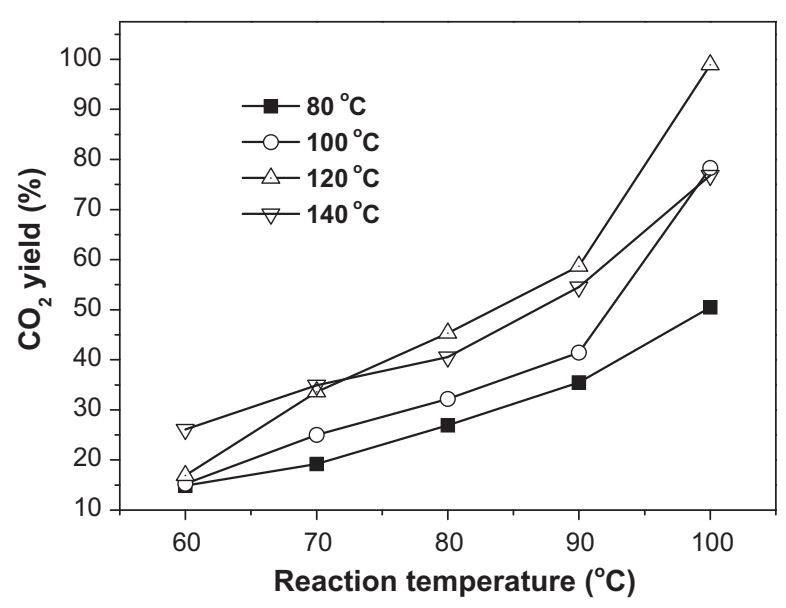

Fig. 6. HCHO conversions on manganese oxide catalysts prepared at varied temperatures. Catalyst: $100 \mathrm{mg}$, space velocity: $30,000 \mathrm{~mL} /\left(g_{\text {cat }} \mathrm{h}\right), \mathrm{HCHO}$ 460 ppm, $\mathrm{O}_{2} 21$ vol.\%. enhanced the $\mathrm{HCHO}$ oxidation. All of these results indicate that the morphology, pore size and the surface area of catalysts are among the major factors governing their high catalytic activity. The surface area of BSW-140 is higher than that of BSW-120 though they have similar pore sizes, however, at elevated temperature $\left(>70{ }^{\circ} \mathrm{C}\right)$ the catalytic activity of BSW-140 slightly decreases, which may be due to the presence of nano-octahedral $\mathrm{Mn}_{3} \mathrm{O}_{4}$ in the BSW-140 material.

\subsection{Redox properties}

To further investigate the relationship between chemical property and catalytic activity, the as-prepared manganese oxides were measured using $\mathrm{H}_{2}$-TPR, and the results are showed in Fig. 7. According to the literature [33], the reduction procedure of manganese oxides can be described by three steps:

$\mathrm{MnO}_{2} \rightarrow \mathrm{Mn}_{2} \mathrm{O}_{3} \rightarrow \mathrm{Mn}_{3} \mathrm{O}_{4} \rightarrow \mathrm{MnO}$

Fig. 7 shows that two or three peaks at $250-500{ }^{\circ} \mathrm{C}$ are observed in each $\mathrm{H}_{2}$-TPR profile, depending on the sample, and the corresponding positions are summarized in Table 2 .

The reduction spectrum of BSW-100 is characterized by three peaks at ca. 324,365 and $462{ }^{\circ} \mathrm{C}$. The first sharp peak may correspond to the transformation of $\mathrm{MnO}_{2}$ to $\mathrm{Mn}_{2} \mathrm{O}_{3}$, while the second peak is assigned to the subsequent reduction to $\mathrm{Mn}_{3} \mathrm{O}_{4}$. The third peak (highest temperature peak, maximum near $462{ }^{\circ} \mathrm{C}$ ) is less intense but broad, and attributed to the reduction of $\mathrm{Mn}_{3} \mathrm{O}_{4}$ to $\mathrm{MnO}$.

A similar reduction pattern was observed for the BSW-120 sample, but the peak positions shift to lower temperatures, indicating higher reducibility. In addition, the intensities of reduction peaks are much higher in comparison to BSW-100, showing higher relative amounts of reducible species (thus, more reactive species and higher reactivity). However, when the synthesis temperature of the sample is increased to $140{ }^{\circ} \mathrm{C}$, the temperatures of three reduction peaks slightly shift to higher temperatures (see Table 2 ), meaning lower reducibility.

The $\mathrm{H}_{2}$-TPR spectrum of $\mathrm{BSW}-80$ is different from the others. Just two major peaks at ca. $308^{\circ} \mathrm{C}$ and $424^{\circ} \mathrm{C}$ were observed. The low temperature peak represents the reduction of $\mathrm{MnO}_{2}$ to $\mathrm{Mn}_{2} \mathrm{O}_{3}$. The broad high temperature peak with a maximum at ca. $424{ }^{\circ} \mathrm{C}$ may be deconvoluted into two overlapping components, indicating that the intermediate reduction from $\mathrm{Mn}_{2} \mathrm{O}_{3}$ to $\mathrm{Mn}_{3} \mathrm{O}_{4}$

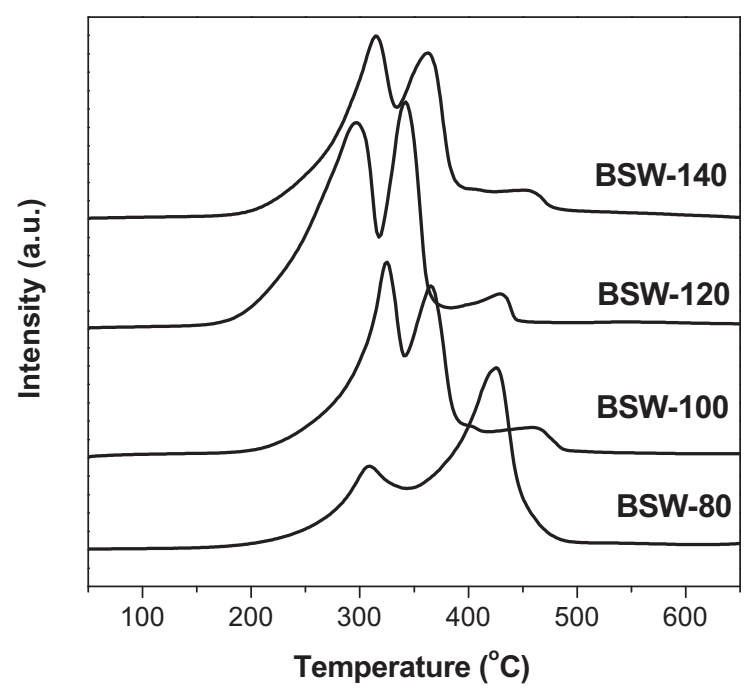

Fig. 7. $\mathrm{H}_{2}$-TPR profiles of varied manganese oxide catalysts. 
Table 2

Peak positions $\left({ }^{\circ} \mathrm{C}\right)$ of the $\mathrm{H}_{2}$-TPR spectra for the manganese oxide catalysts.

\begin{tabular}{llll}
\hline Sample & Peak I $^{\mathrm{a}}$ & Peak II $^{\mathrm{b}}$ & Peak III $^{\mathrm{c}}$ \\
\hline BSW-80 & 308 & 424 & \\
BSW-100 & 324 & 365 & 462 \\
BSW-120 & 296 & 342 & 432 \\
BSW-140 & 314 & 362 & 460 \\
\hline
\end{tabular}

a The first reduction peak in each $\mathrm{H}_{2}$-TPR profile

b The second reduction peak in each $\mathrm{H}_{2}$-TPR profile.

c The third reduction peak in each $\mathrm{H}_{2}$-TPR profile.

may finish at a relatively high temperature, and thus the corresponding component overlaps with the component ascribed to the reduction of $\mathrm{Mn}_{3} \mathrm{O}_{4}$ to $\mathrm{MnO}$.

As far as we know, the surface redox property of catalyst profoundly influences heterogeneous catalytic reactions because the reactions mainly occur on the catalyst surface. Kapteijn et al. [33] reported that manganese oxide with a lower onset temperature of reduction showed a higher catalytic activity in the NO reduction by $\mathrm{NH}_{3}$. Chen et al. [34] found that the facile reduction feature of manganese oxide implied the easy activation for $\mathrm{CO}$ oxidation. It is clear that high surface reducibility results in high catalytic activity in that the reduction of surface species frequently occurs at relatively low temperatures.

In the current work, the order of reducibility of all samples derived from the low temperature reduction peak is BSW-120> BSW-80 > BSW-140>BSW-100, similar to the order of catalytic activity (BSW-120 > BSW-140 > BSW-100 > BSW-80) except BSW80. The $\mathrm{H}_{2}$-TPR results indicate that though the first peak reduction temperature of BSW-80 is lower than those of BSW-140 and BSW100 , its intensity is much lower, meaning consequently lower relative amounts of surface reactive species and lower reactivity. Meanwhile, as compared to BSW-140 and BSW-100, BSW-80 has worse pore structure and lower surface area, all of which leads to its lower catalytic activity for the HCHO oxidation.

It is clear that the catalytic activity of birnessite manganese oxides for HCHO oxidation is influenced by a combination of factors including morphology, surface area and surface reducibility. High surface area and high reducibility increase the catalytic activity of the synthesized manganese oxides, while poor pore structure has the opposite effect. The optimal catalyst obtained is BSW-120.

\section{Conclusion}

We have demonstrated a facile route to synthesizing birnessite manganese oxides in a microemulsion system. The morphology, crystal phase, surface area, pore size, and even the chemical property of manganese oxides were tailorable via controlling the synthesis temperature. By this method, porous birnessite materials were obtained with very high specific surface areas ranging from 138 to $154 \mathrm{~m}^{2} / \mathrm{g}$. This strategy can be extended to the preparation of other useful manganese oxide nanomaterials. The catalytic activity of obtained birnessite manganese oxides for HCHO oxidation is excellent, and is highly dependent on their morphology, surface area and surface reducibility. The most active catalyst
(BSW-120) could completely oxidize $\mathrm{HCHO}$ into $\mathrm{CO}_{2}$ and $\mathrm{H}_{2} \mathrm{O}$ at $100{ }^{\circ} \mathrm{C}$. This value is significantly superior to those of previously reported manganese oxides under similar conditions.

\section{Acknowledgements}

This work was supported by the National Natural Science Foundation of China (Grant 20871118, 21007076), the Knowledge Innovation Program of the Chinese Academy of Sciences (CAS) (Grant KSCX2-YW-G-059), and the National Basic Research Program of China (Grant 2010CB934103).

\section{References}

[1] J. Xu, T. White, P. Li, C. He, Y.F. Han, J. Am. Chem. Soc. 132 (2010) $13172-13173$.

[2] Y. Shen, X. Yang, Y. Wang, Y. Zhang, H. Zhu, L. Gao, M. Jia, Appl. Catal., B 79 (2008) 142-148.

[3] J.Q. Torres, J.M. Giraudon, J.F. Lamonier, Catal. Today 176 (2011) 277-280.

[4] T. Kawai, C. Ohtsuki, M. Kamitakahara, M. Tanihara, T. Miyazaki, Y. Sakaguchi, S. Konagaya, Environ. Sci. Technol. 40 (2006) 4281-4285.

[5] A. Montoya, B.S. Haynes, J. Phys. Chem. A 113 (2009) 8125-8131.

[6] T. Noguchi, A. Fujishima, P. Sawunyama, K. Hashimoto, Environ. Sci. Technol. 32 (1998) 3831-3833.

[7] Y. Wen, X. Tang, J. Li, J. Hao, L. Wei, X. Tang, Catal. Commun. 10 (2009) 1157 1160.

[8] C. Zhang, H. He, K. Tanaka, Appl. Catal. B 65 (2006) 37-43.

[9] J. Zhang, Y. Jin, C. Li, Y. Shen, L. Han, Z. Hu, X. Di, Z. Liu, Appl. Catal. B 91 (2009) $11-20$.

[10] N. An, Q. Yu, G. Liu, S. Li, M. Jia, W. Zhang, J. Hazard. Mater. 186 (2011) 1392 1397.

[11] T. Chen, H. Dou, X. Li, X. Tang, J. Li, J. Hao, Micropor. Mesopor. Mater. 122 (2009) 270-274

[12] I. Atribak, A. Bueno-Lopez, A. Garcia-Garcia, P. Navarro, D. Frias, M. Montes Appl. Catal. B 93 (2010) 267-273.

[13] M. Nakayama, M. Shamoto, A. Kamimura, Chem. Mater. 22 (2010) 5887-5894.

[14] S.L. Brock, N. Duan, Z.R. Tian, O. Giraldo, H. Zhou, S.L. Suib, Chem. Mater. 10 (1998) 2619-2628.

[15] H. Chen, J. He, C. Zhang, H. He, J. Phys. Chem. C 111 (2007) 18033-18038.

[16] M.A. Cheney, R. Jose, A. Banerjee, P.K. Bhowmik, S. Qian, J.M. Okoh, J. Nanomater. (2009), ID 940462

[17] J. Yan, Z. Fan, T. Wei, W. Qian, M. Zhang, F. Wei, Carbon 48 (2010) 3825-3833.

[18] P. Yu, X. Zhang, Y. Chen, Y. Ma, Mater. Lett. 64 (2010) 1480-1482.

[19] Y. Cho, S. Yang, Y. Kim, S. Komarneni, S. Kim, J. Porous. Mater. 18 (2011) 125 131.

[20] H. Tian, J. He, L. Liu, Adv. Mater. Res. 181-182 (2011) 485-488.

[21] H. Chen, J. He, J. Phys. Chem. C 112 (2008) 17540-17545.

[22] L. Xiao, Y. Zhao, J. Yin, L. Zhang, Chem. Eur. J. 15 (2009) 9442-9450.

[23] H.T. Zhu, J. Luo, H.X. Yang, J.K. Liang, G.H. Rao, J.B. Li, Z.M. Du, J. Phys. Chem. C 112 (2008) 17089-17094.

[24] L. Zhang, Q. Zhou, Z. Liu, X. Hou, Y. Li, Y. Lv, Chem. Mater. 21 (2009) 50665071.

[25] R.G. Laughlin, The Aqueous Phase Behavior of Surfactants, Academic Press, London, 1994.

[26] H. Xu, Q. Liu, Spectrochim. Acta Pt. A 70 (2008) 243-246.

[27] Z.X. Liang, J.H. Li, Functional Emulsifier and Emulsion, China Light Industry Press, Beijing, 2000

[28] S.J. Gregg, K.S.W. Sing, Adsorption, Surface Area and Porosity, second ed., Academic Press Inc., London, 1982.

[29] H. Tian, J.He, X. Zhang, L. Zhou, D. Wang, Micropor. Mesopor. Mater. 138 (2011) 118-122.

[30] X. Tang, J. Li, J. Hao, Catal. Commun. 11 (2010) 871-875.

[31] Y. Xia, H. Dai, L. Zhang, J. Deng, H. He, C.T. Au, Appl. Catal. B 100 (2010) 229237.

[32] Z. Abbasi, M. Haghighi, E. Fatehifar, S. Saedy, J. Hazard. Mater. 186 (2011) 1445-1454.

[33] F. Kapteijn, L. Singoredjo, A. Andreini, Appl. Catal. B 3 (1994) 173-189.

[34] J. Chen, J. Li, H. Li, X. Huang, W. Shen, Micropor. Mesopor. Mater. 116 (2008) 586-592. 